ARTICLE

\title{
Direct Numerical Simulation of Turbulent Channel Flow with Deformed Bubbles
}

\author{
Yoshinobu YAMAMOTO* and Tomoaki KUNUGI \\ Kyoto University, Yoshida, Sakyo, Kyoto, 606-8501, Japan
}

\begin{abstract}
In this study, the direct numerical simulation of a fully-developed turbulent channel flow with deformed bubbles were conducted by means of the refined MARS method, turbulent Reynolds number 150, and Bubble Reynolds number 120. As the results, large-scale wake motions were observed round the bubbles. At the bubble located region, mean velocity was degreased and turbulent intensities and Reynolds shear stress were increased by the effects of the large-scale wake motions round bubbles. On the other hands, near wall region, bubbles might effect on the flow laminarlize and drag reduction.

Two types of drag coefficient of bubble were estimated from the accelerated velocity of bubble and correlation equation as a function of Particle Reynolds number. Empirical correlation equation might be overestimated the drag effects in this Particle Reynolds number range.
\end{abstract}

KEYWORDS: DNS, bubbly flow, turbulent shear flow, drag friction, gas entrainment

\section{Introduction}

Turbulent flows with bubbles are very often found in engineering devices such as a light-water nuclear reactor and a chemical reactor. Therefore, both experimental and numerical investigations have been conducted, extensively. In the view points of understanding coherent structures and turbulent statistics behaviors, the DNS (Direct Numerical Simulation) is expected to have advantages over the experimental approaches.

Recently, DNS of the turbulent shear flows with deformability bubbles have been conducted by Kawamura and Kodama, ${ }^{1)}$ Lu et al., ${ }^{2)}$ and Lu and Tryggvason, ${ }^{3)}$ by means of the front-tracking method. These previous DNSs focused on interaction between turbulent structures near wall and bubbles to investigate friction drag reduction effects on the wall.

In the view points of the computational model ${ }^{4)}$ for the gas and bubble behavior in the primary coolant system of a sodium-cooled Fast Reactor, upgrading of not only the wall drag coefficient but also the drag coefficient acted on the deformable bubbles is indispensable.

In this study, we applied the MARS method ${ }^{5)}$ as the direct numerical investigation scheme for the drag coefficient acted on the deformable bubbles in the turbulent shear flows and the DNS database of a fully-developed turbulent channel flow with bubbles were established.

\section{Numerical Method}

\section{MARS Method}

Numerical procedure was based on the MARS method;5) the governing equations are consisted of Navier-Stokes equations, continuity equation and transport equation of a volume fraction function (VOF) $F$. Regarding the discretiza-

*Corresponding author, E-mail:yyama@nucleng.kyoto-u.ac.jp

(C) 2011 Atomic Energy Society of Japan, All Rights Reserved. tion of the velocity fields on the Cartesian coordinate system, the second-order scheme for the spatial differencing terms is used on the staggered grid system. The physical problem treated here is the motion of two Newtonian incompressible fluids allowed the interface deformation between them.

To adapt the MARS method for the DNS of turbulent bubbly flows, two improvements of the original MARS method were conducted. One was the refinement of the calculation of a surface tension force and another was the upgrading the time integration schemes.

In the original MARS method, the surface tension forces ( $: F_{v i}$, the $i$-th components of surface tension force, $i=1,2,3$ ) according to the CSF model, ${ }^{6}$ are defined by the following equations:

$$
\begin{aligned}
& F_{v i}=\sigma \kappa \frac{\partial F}{\partial x_{i}} \frac{\langle\rho\rangle}{\bar{\rho}}, \kappa=-\frac{\partial n_{i}}{\partial x_{i}}, \\
& \langle\rho\rangle=\sum\left(F_{m} \rho_{m}\right), \quad \bar{\rho}=\left(\rho_{g}+\rho_{l}\right) / 2,
\end{aligned}
$$

where $\rho, \sigma, \kappa$, and $n_{\mathrm{i}}$ denote density, coefficient of surface tension, interface curvature, and $i$-th components of the interface normal vector, respectively.

In this study, to refine the calculation of the surface tension acted on the bubbles, a kind of the level-set function $(: \phi)^{7)}$ estimated by using the VOF function, was used for the calculation of the surface tension forces, as following, ${ }^{8)}$

$$
\begin{aligned}
& F \Rightarrow \nabla|\phi|=1, \quad \phi(x, y, z) \begin{cases}>0 & \text { Liquid } \\
=0 & \text { Interface } \\
<0 & \text { Gas }\end{cases} \\
& F v_{i}=\sigma \kappa \frac{\partial \phi}{\partial x_{i}} \frac{\langle\rho\rangle}{\bar{\rho}}, \kappa=-\frac{\partial \phi}{\partial x_{i}} .
\end{aligned}
$$

A second improvement was adapting the high-accuracy time integration schemes for evaluating the acceleration of bub- 


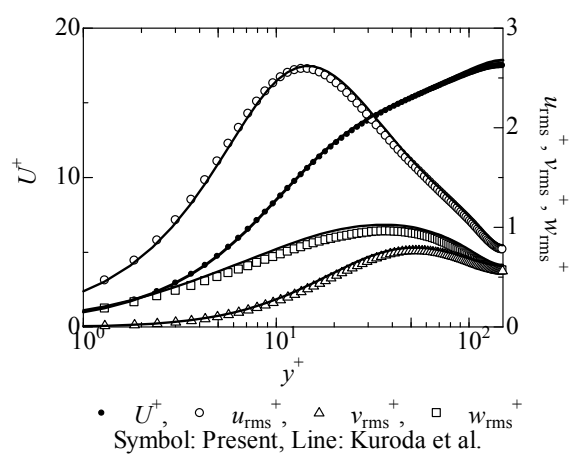

(a)

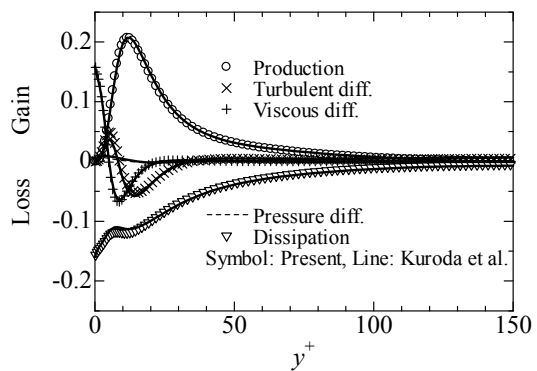

Fig. 1 Verifications of the modified MARS method compared with results by Kuroda et al., (a) mean velocity and turbulent intensities, and (b)budget of turbulent kinetic energy.

bles in the turbulent shear flows. In the original MARS method, the 1st-order implicit scheme was used but low-storage 3rd-order Runge-Kutta scheme for convection terms, Crank-Nicolson scheme for viscous terms and Euler Implicit scheme for Pressure terms were implemented. As the results, we can ensure the over second-order accurate time discretization.

\section{Single-Phase Turbulent Channel Flow Simulation}

The accuracy of the modified MARS method was examined for the single-phase turbulent channel flow at a turbulent Reynolds number 150 based on friction velocity channel half height and kinetic viscosity. Computation was carried out using $72 \times 182 \times 72$ grids for the streamwise, vertical, and spanwise directions respectively. Grid resolution normalized by the friction velocity and the kinetic viscosity was $16.7\left(\Delta x^{+}\right), 0.5-2.0\left(\Delta y^{+}\right)$, and $8.3\left(\Delta z^{+}\right)$for streamwise, vertical, and spanwise direction, respectively. Computational time step was adjusted correspond to the maximum CFL was 1.0. Figure 1(a) shows the mean velocity and turbulent intensity profiles; the budget of the turbulent kinetic energy was also shown in Fig. 1(b). Despite the large-time steps in the present case, not only low order statistics (: mean velocity and turbulent intensities) but also high order statistics (: energy dissipation and turbulent diffusion) agreed well with the DNS results of Kuroda et al. ${ }^{9)}$

\section{Rising Bubbles Simulation}

To check the upgrading of the time integration and surface tension forces, two rising bubble simulation was also carried out. Computational geometry, example of the time series behaviors of bubbles and numerical condition, were

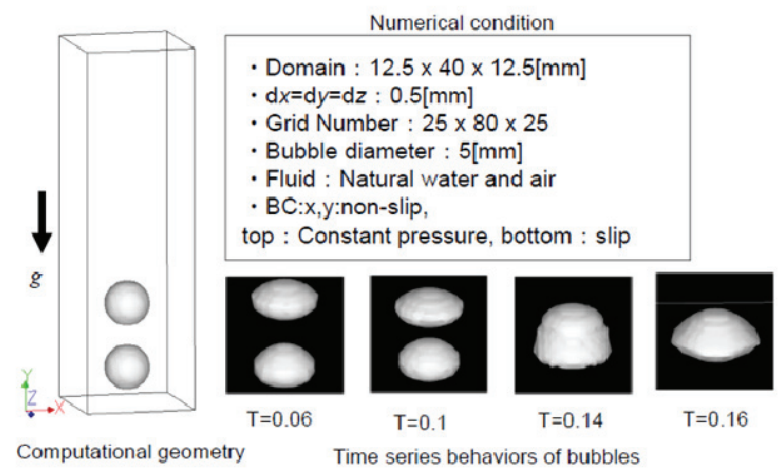

Fig. 2 Rising bubbles simulation, flow geometry, numerical condition, and time series of bubble behaviors

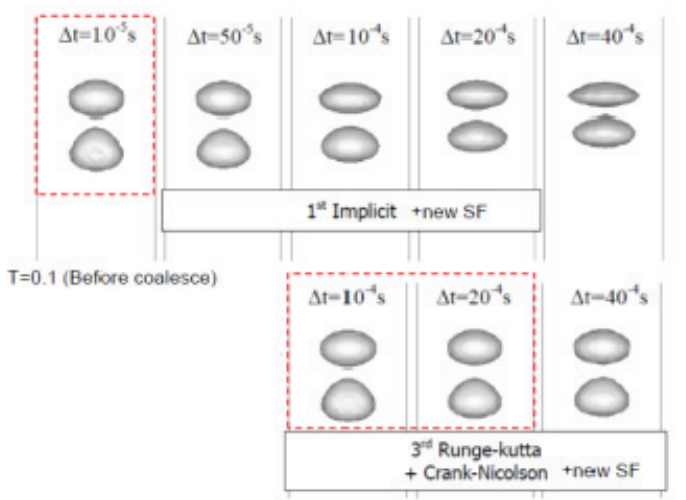

Fig. 3 Comparison of the bubble shapes at $T=0.1$ (before coalescing) in case of the 1st order time integration schemes and the high-order schemes with various time steps

summarized in Fig. 2. In this simulation, bubbles were rising driven by buoyancy and the underside bubble caught the upper bubble and they coalesced into the single bubble as shown in Fig. 2.

Figure 3 shows the comparison of the bubble shapes before coalescing time $T=0.1$. The upper figures were calculated by using the 1 st order time integration schemes and the lower figures were calculated by the high-order schemes, with various time step intervals. Using the present new calculation for surface tension forces, unphysical behaviors caused form the lacks of the surface tension accuracy cannot be observed in both schemes. In cases of the 1st-order time integration scheme $\left(\Delta \mathrm{t}=10^{-5}\right)$ and the high-order scheme $\left(\Delta \mathrm{t}=10^{-4}, 20^{-4}\right.$, and $\left.40^{-4}\right)$, rising bubble velocities were corresponding to others within an uncertainty of about $6 \%$ and bubble shapes gives close agreement with each other; These indicate that the high-order scheme has more than tenfold advantage in the time step interval compared with the 1st-order scheme.

\section{Numerical Condition of a Turbulent Channel Flow with Bubbles}

Figure 4 shows flow geometry and coordinate systems. Numerical condition was tabled in Table 1. In this study, 


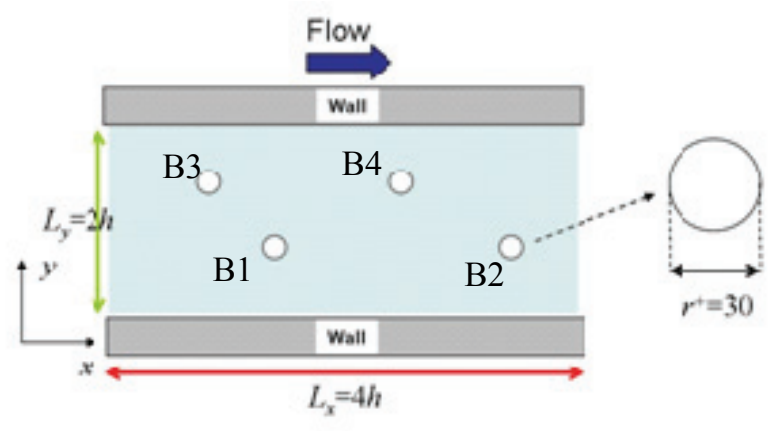

Fig. 4 Flow geometry and coordinate system

Table 1 Numerical condition

\begin{tabular}{ccc}
\hline CASE & CASE1 & CASE2 \\
\hline $\operatorname{Re}_{\tau}$ & $150\left(=h^{+}\right)$ & \\
\hline$r^{+}$ & $30(=0.2 h)$ \\
\hline$\rho_{w} / \rho_{\mathrm{g}}$ & \multicolumn{2}{c}{842.1} \\
\hline Domain, \\
$L_{x} L_{y}, L_{z}$ \\
\hline $\begin{array}{c}\text { Grid number, } \\
N_{x}, N_{v}, N_{z}\end{array}$ & $150,134,50$ \\
\hline Resolution, & $300,182,100$ \\
$\Delta x^{+}, \Delta y^{+}, \Delta z^{+}$ & $4.0,0.5-4.0,4.0$ & $2.0,0.5-2.0,2.0$ \\
\hline$T_{0}^{+}$ & & \\
\hline
\end{tabular}

$\mathrm{Re}_{\tau}=u_{\tau} h / v_{w}$ : Turbulent Reynolds number,

$u_{\tau}$ : Friction velocity at wall, $2 h$ : water depth,

$v_{w}$ : Kinetic viscosity of water, $r$ : Initial radius of bubbles, $\rho_{w}$ : Density of water, $\rho_{\mathrm{g}}$ :Density of air,

$L_{x}, L_{y}, L_{z}$ : Computational domain,

$N_{x}, N_{y}, N_{z}$ : Grid number,

$\Delta x^{+}, \Delta y^{+}, \Delta z^{+}:$Grid resolution for stream $(x)$-, vertical $(y)-$, and span $(z)$ - wise directions, respectively.

Super-script + denotes the nondimensional quantities normalized by friction velocity and kinetic viscosity used Reynolds number definition.

$T_{0}^{+}$: Time integration length from initial condition to ful-

ly-developed status.

small computational domain as same as the minimum flow units $^{10)}$ was employed. Thermal properties at natural air-water system were adapted. As the initial velocity fields, a fully developed single-phase turbulent channel flow data was used and 4 bubbles which diameter was $1 \mathrm{~mm}$ were entrained in channel center region. Thus, void fraction was only $0.15 \%$. To obtain the fully-developed status, gravity effect was ignored and the streamwise constant pressure gradient was forced on the water phase. Non-slip at the walls and periodic conditions for the stream and spanwise directions were imposed for the boundary conditions.

From the initial condition, time integration was conducted during 400 non-dimensional times $\left(: T_{0}^{+}=T_{0} u_{\tau}^{2} / v_{w}, T_{0}\right.$ : time, $u_{\tau}$ : friction velocity at the wall, $v_{w}$ : kinetic viscosity of the water phase), to obtained the fully-developed status. Then, it was confirmed that flow fields reached the fully-developed status After that, $T^{+}=1,200$ non-dimensional time integration was conducted to obtain the mean and statistics data.

\section{Numerical Results}

\section{Effect of Grid Resolution}

Figures 5(a) and (b) show the instantaneous turbulent velocity vector plots round bubbles in CASE1 (Fig. 5(a)) and CASE2 (Fig. 5(b)). Here, turbulent velocity denotes the velocity difference from its mean value. Large-scale wake motions round the bubbles were observed. These wake motions round bubbles were no much difference between CASE1 and CASE2.

Figure 5(c) shows the turbulent intensity profiles in CASE1 and 2. Note that these turbulent intensities were averaged without the distinction between air and water phases. Near channel center region $\left(y^{+}>50\right)$, these differences of turbulent intensities were indifference. On the other hands, there were the slightly differences near wall region $\left(y^{+}<30\right)$. It was guessed that these differences near wall region were not depended on the grid resolution but the bubble detention time in this region. Therefore, to discuss the grid resolution effects of bubbly flows on near wall turbulent structures, many numbers of bubbles or very long time integration might be required to ignore the effects of the bubble detention time.

However, at least, we can confirm that the grid resolution effect on turbulent structures near channel center was small. Hereafter, to discuss the drag coefficient acted on the deformable bubbles in the channel center region and the results of CASE 1 were used as the DNS database.

\section{Bubble Behavior and Distribution}

Figure 6 shows instantaneous bubble behaviors with streamwise turbulent velocity contour plots in time periods $T^{+}=230-250$. After flow have reached the fully-developed status, large- wake motion round bubbles were constantly observed and bubble deformation scale was about $7 \%$ of the initial bubble diameter.

In this study, bubbles were labeled as B1, B2, B3, and B4 to distinguish from others as shown in Fig. 4. Figure 7 shows the distribution of the mean volume-rate function of bubble B1 and B3. Vertical motions of bubble B1 and B3 were confined in the channel center region and fluctuations of vertical bubble positions were within 20 in wall units, during the whole time integration length $\left(T^{+}=1,200\right)$.

On the other hands, after $T^{+}=600$, the bubble labeled as B2 moved to the near wall region and interaction between this bubble and near wall streaky structures were observed as shown in Fig. 8. In Fig. 8(a), near wall region, bubble might be led to the low-pressure regions of the high-speed streaky structures. Wakes behind the bubble were observed both in Figs. 8(b) and (c), but the high-speed region in front of the bubble was diminished as shown in Fig. 8(c). These might indicate that bubbles disturb the near wall coherent turbulent motion. ${ }^{10)}$

\section{Mean Velocity and Statistics}

Water-phase mean velocity profile was shown in Fig. 9(a). Mean velocity at the channel center region were decreased compared with the single-phase flow; water-phase discharge was also decreased. This indicates that bubbles works as the 


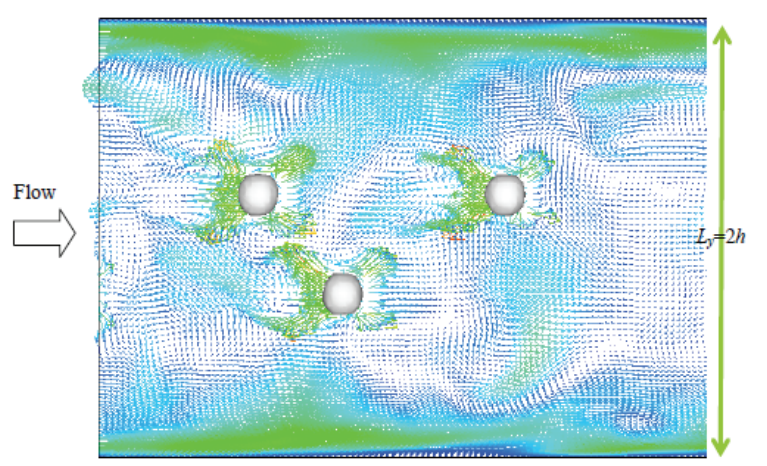

(a)

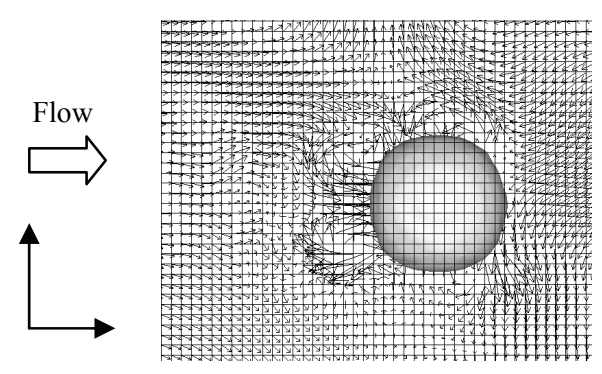

Side view

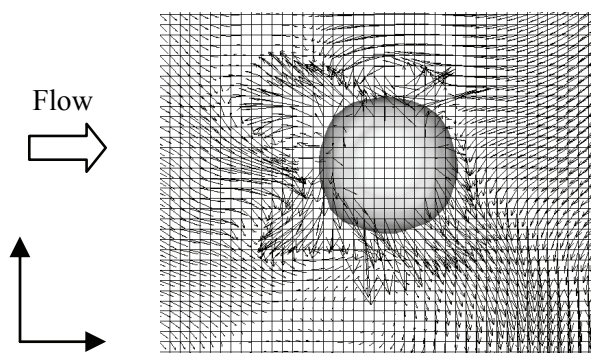

Top view

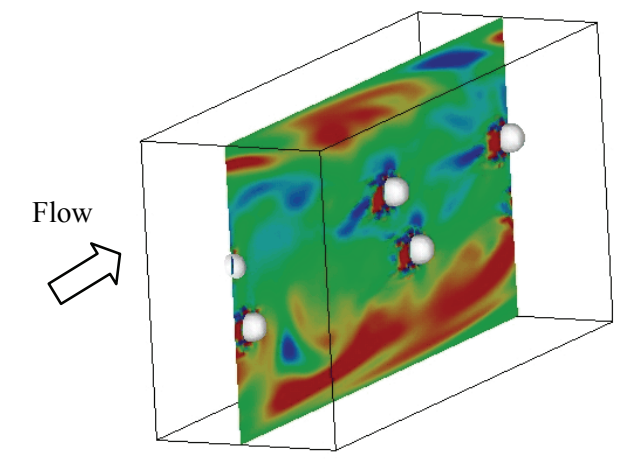

(a)

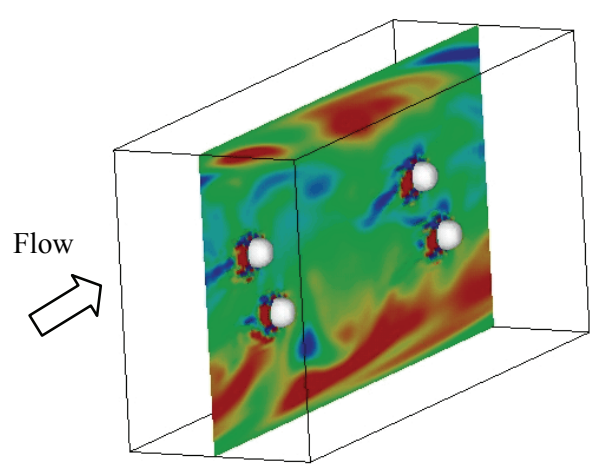

(b)

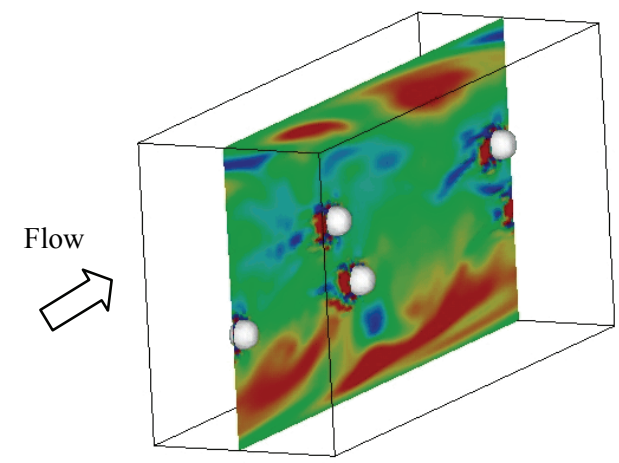

(c)

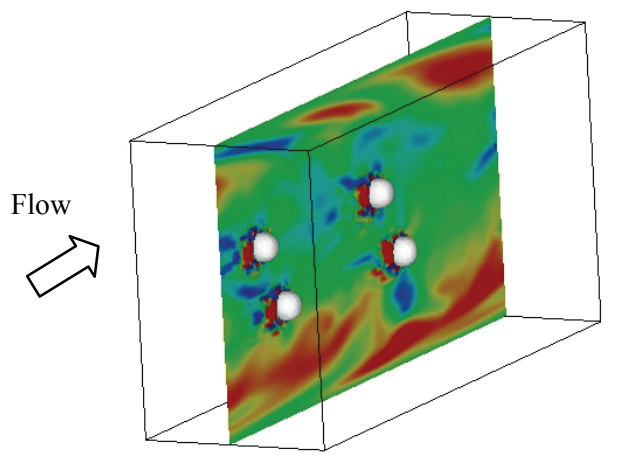

(d)
Fig. 5 Grid resolution dependency, (a) instantaneous turbulent velocity vector plots, side view, CASE1, (b) instantaneous turbulent velocity vector plots, side view and top view, CASE2, (c) turbulent intensity profiles, CASE1 and 2.
Fig. 6 Instantaneous bubble behaviours with streamwise turbulent velocity contour plots, bird view, CASE1, -3.5 (blue) $<u^{+}<$ 3.5 (red), (a) $T^{+}=232.02$, (b) $T^{+}=238.03$, (c) $T^{+}=244.12$, and (d) $T^{+}=250.01$. 


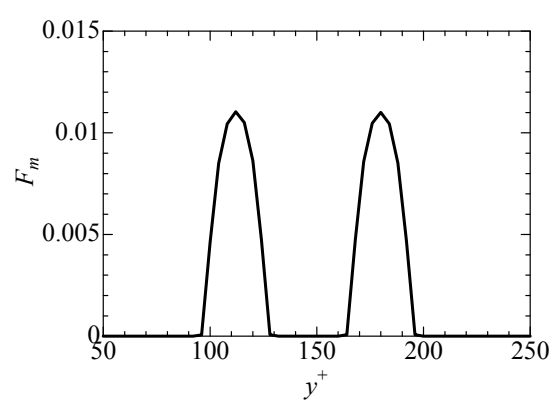

Fig. 7 Mean volume-rate function distribution of bubble B1 and B2

(a)

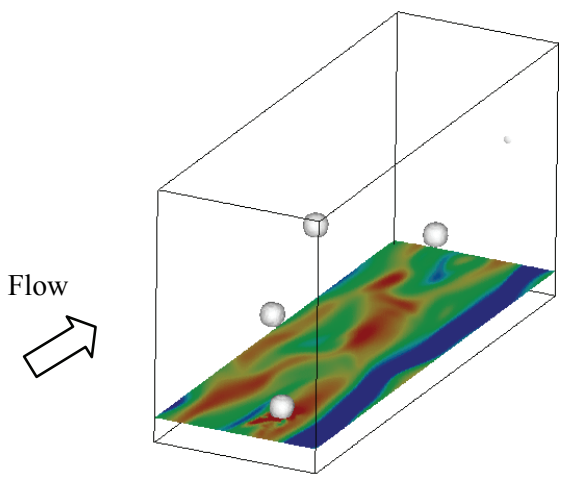

(b)
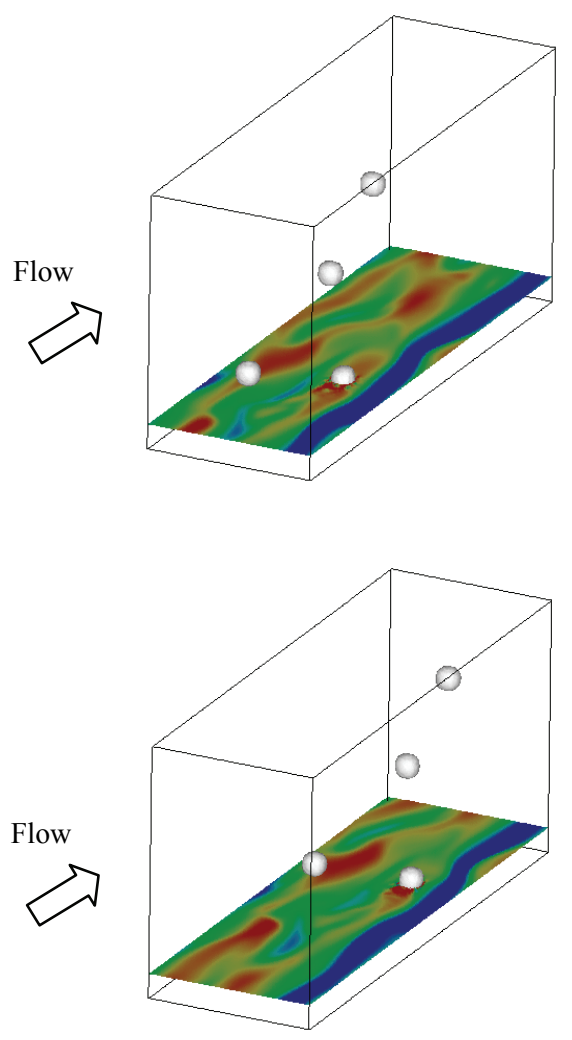

(c)

Fig. 8 Interaction between bubbles and high-speed streaky structures, streamwise velocity contour, -3.5 (blue) $<u^{+}<$ $3.5(\mathrm{red})$ at $y^{+}=28$, (a) $T^{+}=621.08$, (b) $T^{+}=632.10$, and (c) $T^{+}=$ 667.27 . flow resistance in this flow condition. Figures 9(b) and (c) show the water-phase turbulent intensities and Reynolds shear stress profiles. Near channel center, turbulent intensities and Reynolds shear stress were increased compared with in case of the single-phase flow. Increase of the turbulence is caused from the large-scale wake motions round bubbles as shown in Fig. 5. On the other hands, near wall region, peak position of the streamwise turbulent intensity was slightly shifted to the channel center and the Reynolds stress near wall was decreased. Consequently, it seems that bubbles act on the flow laminarlize and the drag reduction near the wall region.

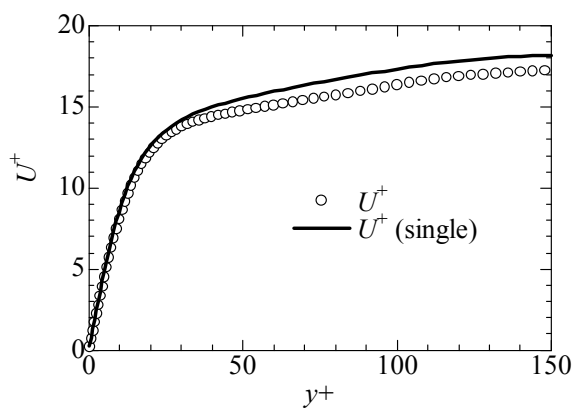

(a)

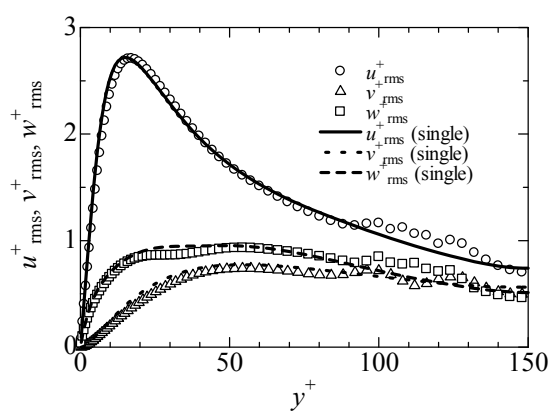

(b)

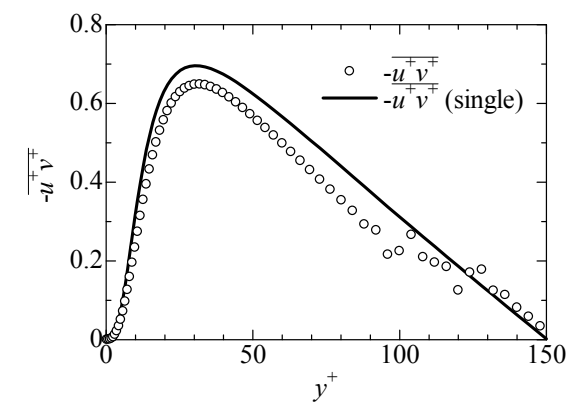

(c)

Fig. 9 Mean velocity and statistics profiles, CASE1, (a) mean velocity, (b) turbulent intensities, and (c) Reynolds stress. 


\section{Particle Reynolds Number and Drag Coefficient}

In this section, we discussed the drag coefficient acted on the deformed bubbles labeled as B1 and B3 in CASE1. These bubbles were confined in the channel center region, during the whole time integration length $\left(T^{+}=1,200\right)$.

The drag coefficient acted on the bubbles was defined as:

$$
\begin{aligned}
& \frac{d^{2} X}{d t^{2}}=F_{D} / m, \\
& F_{D}=-\frac{C_{D}}{2} \rho_{a}\left(u_{p}-u_{b}\right)\left|u_{p}-u_{b}\right| \frac{\pi D^{2}}{4},
\end{aligned}
$$

where $X$ is the position of bubble center, $F_{D}$ is the forces acting on bubbles, $m$ is the mass of bubble, $C_{D}$ is the drag coefficient of bubble, $u_{D}$ is the bubble velocity, $u_{b}$ is the water-phase velocity and $D$ is the initial bubble diameter. From Eqs. (4) and (5), drag coefficient of bubble can be estimated by the following equation,

$$
C_{D}=\frac{8 D a}{6\left|u_{p}-u_{b}\right|^{2}},
$$

where $a\left(=d^{2} X / d t^{2}\right)$ is the bubble acceleration. If the particle Reynolds number $\left(\operatorname{Re}_{\mathrm{p}}\right)$ was given and bubble deformation can be ignored, the drag coefficient was also estimated by using the following empirical correlation equation.

$$
\begin{aligned}
C_{D} & =\left(0.55+4.8 / \sqrt{\operatorname{Re}_{p}}\right)^{2}, 1<\operatorname{Re}_{p} \leq 1000, \\
\operatorname{Re}_{p} & =\frac{\left|u_{p}-u_{b}\right| D}{v},
\end{aligned}
$$

Particle Reynolds number was directly obtained by DNS data as shown in Fig. 10, and the two drag coefficients of bubble were estimated by using Eqs. (6) and (7).

Figure 10 shows the time series of the instantaneous particle Reynolds number. Instantaneous particle Reynolds number changed within the range from 110 to 135 and its range was within the assumption of Eq. (7). Averaged particle Reynolds number was 122 .

Figure 11 shows the time series of the drag coefficient of bubble. In Fig. 11(a), the local mean water-phase velocity at the bubble center position was used as the water-phase velocity $\left(: u_{f}=u_{b}\right)$. On the other hands, the bulk water-phase velocity $\left(: u_{b}\right)$ was used in Fig. 11(b).

In Fig. 11(a), the drag coefficients estimated by Eqs. (6) and (7) were consisted well. However, in Fig. 11(b), the drag coefficient (shown as the line) estimated by the empirical correlation equation (7) were overestimated one (shown as broken line) evaluated by Eq. (6). This is caused from the increase of the relative bubble velocity.

If the relative bubble velocity was estimated by the bulk velocity, interactions between bubbles and the large-scale wake motions seemed to be ignored and under estimated the drag effects.

In spite of the definition of water-phase velocity, drag coefficient estimated by Eq. (6) was below the results estimated by the correlation equation (7). This implies that empirical correlation equation might be overestimated the drag effects in this Particle Reynolds number range.

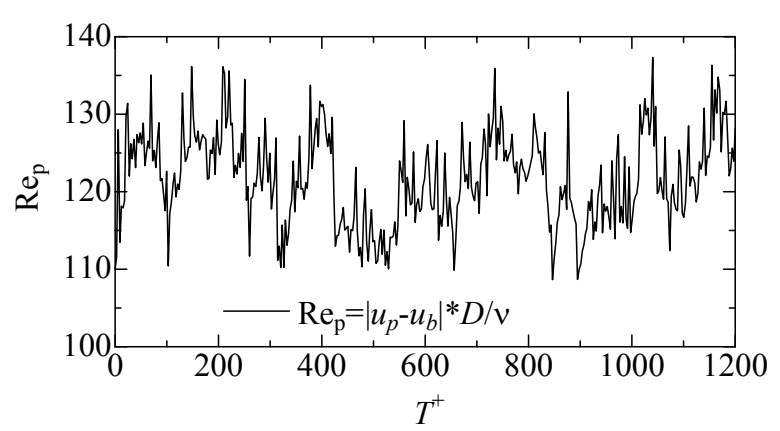

Fig. 10 Time series of instantaneous particle Reynolds number

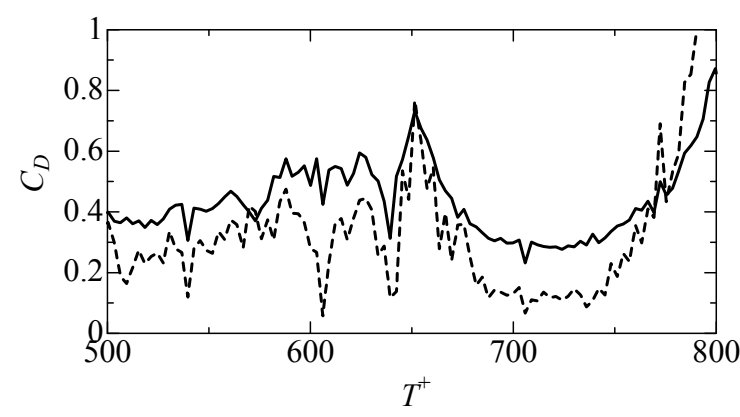

$-C_{D}=\left(0.55+4.8 / R e_{p}^{0.5}\right)^{2}, R e_{p}=\left|u_{p}-u_{f}\right|^{*} D / v$
$--C_{D}=8 / 6^{*} D^{*} a /\left|u_{p}-u_{f}\right|^{2}$
$u_{p}:$ Bubble velocity
$u_{f}$ : Water-velocity at bubble position

(a)

$$
\begin{aligned}
& u_{p}: \text { Bubble velocity } \\
& u_{f} \text { : Water-velocity at bubble position }
\end{aligned}
$$

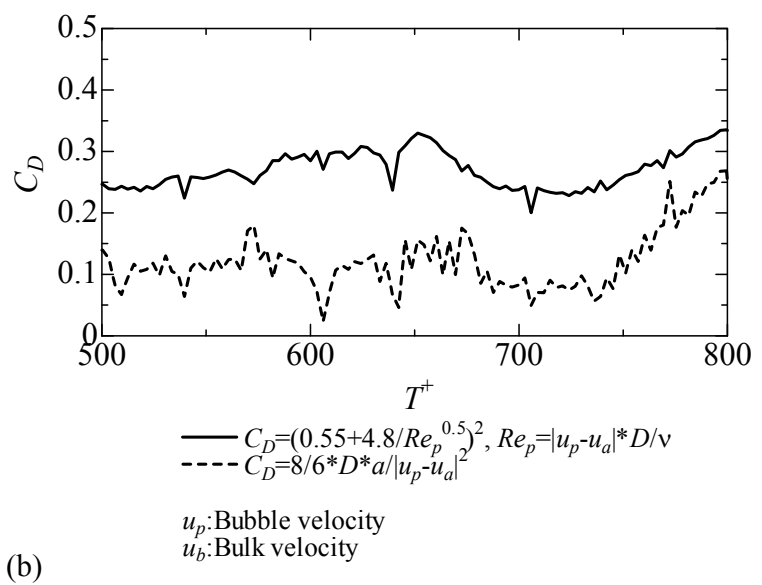

Fig. 11 Time series of drag coefficient of bubble, (a) estimated by using the local mean water-phase velocity, and (b) estimated by using the bulk water-phase velocity.

\section{Conclusion}

In this study, the direct numerical simulations of a fully-developed turbulent channel flow with bubbles were conducted by means of the refined MARS method. DNS database such as the mean velocity, turbulent statistics and drag coefficient of deformed bubble were obtained. Main results are summarized:

1) In this flow condition, average particle Reynolds number was about 120 , large-scale wake motions effected by bubbles were observed. 
2) At the bubble located region, mean velocity was degreased and turbulent intensities and Reynolds shear stress were increased by the effects of the large-scale wake motions round bubbles.

3) The peak position of the streamwise turbulent intensity was slightly shifted to channel center and Reynolds stress near wall was decreased; near wall region, bubbles might effect on the flow laminarlize and drag reduction.

4) Empirical correlation equation might be overestimated the drag effects in this Particle Reynolds number range.

\section{Acknowledgment}

A part of the present DNS were conducted by using SX-9 at the Cyber Science Center, Tohoku University, and this the study was supported by the Global COE program "Energy Science in the Age of Global Warming" and a Grant-in-aid for Young Scientists (B), KAKENHI (21760156) MEXT, Japan.

\section{References}

1) T. Kawamura, T. Kodama, "Numerical simulation method to resolve interactions between bubbles and turbulence," Int. J. Heat Fluid Flow, 23, 627-638 (2002).

2) J. Lu, A. Fernandez, G. Tryggvason, "The effect of bubbles on the wall shear in a turbulent channel flow," Phys. Fluid, 17, 095102 (2005).
3) J. Lu, G. Tryggvason, "Effect of Bubble Deformability in Turbulent Bubbly Upflow in a Vertical Channel," Phys. Fluid, 20040701 (2008).

4) A. Yamaguchi, A. Hashimoto, "A Computational Model for Dissolved Gas and Bubble Behavior in the Primary Coolant System of Sodium-Cooled Fast Reactor," The 11th International Topical Meeting on Nuclear Reactor Thermal-Hydraulics (NURETH-11), Avignon, France, Oct. 2-6 (2005).

5) T. Kunugi, "MARS for multiphase calculation," Comput. Fluid Dynam. J., 9, 563-571 (2001).

6) J. U. Brackbill, D. B. Kothe, C. Zemach, "A continuum method for modeling surface tension," J. Comput. Phys., 100, 335 (1992).

7) M. Sussman, P. Smereka, S. Osher, "A Level Set Approch for Computing Solution to Incompressible Two-Phase Flow," J. Comput. Phys., 114, 146-159 (1994).

8) T. Himeno, T. Watanabe, "Numerical analysis of sloshing and wave breaking in a vessel by CIP-LSM," Proc. of APCOM'07 in conjunction with EPMESC XI, Kyoto, 3-6 December, 2007. No. MS7-7-1 (2007) [CD-ROM].

9) A. Kuroda, N. Kasagi, M. Hirata, "Investigation of Dynamical Effects of the Mean Shear Rate on the Wall Turbulence via Direct Numerical Simulation, "27th National Heat Transfer Symposium of Japan, 46-48 (1990).

10) J. Jimenez, P. Moin, "The minimal flow unit in near-wall turbulence," J. Fluid Mech., 225, 213-240 (1991). 